\title{
MEMÓRIA PROCEDIMENTAL E LINGUAGEM: UM ESTUDO COM PESSOAS QUE GAGUEJAM FALANTES DO PORTUGUÊS BRASILEIRO
}

\author{
MEMORIA PROCEDIMENTAL Y EL LENGUAJE: UN ESTUDIO CON PERSONAS QUE TARTAMUDEAN \\ HABLANTES DE PORTUGUÉS BRASILEÑO
} PROCEDURAL MEMORY AND LANGUAGE: A STUDY WITH BRAZILIAN PORTUGUESE STUTTERING
SPEAKERS

\author{
José Ferrari Neto* \\ Débora Vasconcelos Correia \\ Universidade Federal da Paraíba - UFPB, João Pessoa, BR
}

\begin{abstract}
RESUMO: Este artigo apresenta os resultados de uma pesquisa experimental sobre a memória procedimental de pessoas que gaguejam (PQG) e falantes fluentes (FF) do Português Brasileiro (PB), a partir da relação entre o Modelo Pré-Motor Duplo de Alm (2005) e o Modelo Declarativo/Procedimental de Ullman (2001). Lança-se, então, uma hipótese acerca da conexão entre a presença de disfunções mnemônicas e o processamento linguístico das PQG. Os resultados encontrados por meio do Teste ASRT (Alternating Serial Reaction Time) apontaram para uma tendência de comportamento distinto entre os grupos. As PQG evidenciaram um aumento do tempo de reação à medida que se aumentava o número de ciclos (estímulos). O que interpretamos como uma possível dificuldade das PQG na aprendizagem implícita das sequências motoras. Já os FF apresentaram redução do tempo de reação à medida que se aumentava o número de ciclos, demonstrando que a aprendizagem procedimental para este grupo ocorreu de maneira mais rápida.
\end{abstract}

PALAVRAS-CHAVE: psicolinguística experimental; gagueira; memória procedimental; Teste ASRT..

RESUMEN: Este artículo presenta los resultados de una investigación experimental sobre la memoria procedimental de las personas que tartamudean (PQT) y de las personas que hablan con fluidez (PHF) el Portugués de Brasil (PB), a partir de la relación entre el Modelo Pré-Motor, de Alm (2005), y el Modelo Declarativo/Procedimental, de Ullman (2001). Lanza, entonces, una hipótesis sobre la relación entre la presencia de disfunción de memoria y el procesamiento lingüístico de las PQT. Los resultados encontrados por el ASRT apuntan a una conducta distinta entre los grupos. Las PQT mostraron un aumento en el tiempo de reacción a medida que aumentaba el número de ciclos (estímulos), lo que interpretamos como una posible dificultad de las PQG en el aprendizaje implícito de secuencias motoras. Las PHF, a su vez, disminuyeron el tiempo de reacción a medida que aumentaba el número de ciclos, lo que demuestra que el aprendizaje de procedimientos para este grupo se produjo más rápidamente.

PALABRAS CLAVE: psicolingüística experimental; tartamudez; memoria procedimental; Teste ASRT.

ABSTRACT: This paper shows the results of an experimental study about both stutters and fluent speakers's procedural memory in Brazilian Portuguese (BP), from the relation among Alm's Double Pre-Motor Model (ALM, 2005) and Ullmann's Declarative/Procedural Model (ULMANN, 2001). It is proposed a hypothesis about the connection between the presence of mnemonic disfunctions and the stutters's linguistics processing. The results obtained through ASRT Test (Alternating Serial Reaction Time) suggested an increase of reaction times as the number of cycles (stimuli) was augmented. This can be a possible difficulty to implicit learning of motor sequences. On the other hand, the fluent speakers showed a reduction of reaction times as the number of cycles augmented, confirming that, to this group, procedural learning happened in a faster way.

KEYWORDS: experimental psycholinguistics; coreference; stuttering; procedural memory; self-paced reading.

\section{INTRODUÇÃO}

Este artigo tem por objetivo refletir sobre a possibilidade de associação entre a gagueira e a presença de dificuldades na memória procedimental, através da investigação de possíveis diferenças em relação aos falantes fluentes (FF), ambos os grupos nativos do Português Brasileiro (PB). Para isso, estruturamos o presente estudo de acordo com a perspectiva de análise por planos complementares, proposta por Marr $(1982)^{1}$ para as ciências cognitivas, de modo que a sua disposição organizacional apresenta-se distribuída em três níveis, que aqui chamamos de nível neural, procedimental e linguístico.

\footnotetext{
*Emails: joseferrarin@ibest.com.br; fgadebora@gmail.com.

${ }^{1} \mathrm{O}$ estudo de Marr (1982) intitulado "Vision: a computational investigation into the human representation and processing of visual information" propõe uma análise dos sistemas de processamento da informação por meio de planos complementares, também conhecida como a Hipótese dos Três Níveis. Nesse trabalho de Marr especificamente, o sistema estudado foi o da visão. No entanto, pode-se aplicar essa proposta de análise a inúmeros outros
} 
No nível neural apresentamos as razões neurológicas envolvidas na gagueira e que estão atualmente em maior discussão, com ênfase no Modelo Pré-Motor Duplo de Alm (2005), expondo a relação entre este distúrbio da fluência e os núcleos da base. No nível procedimental apontamos a possibilidade de ligação entre uma disfunção da memória procedimental e a gagueira, para isso, tomamos como base o Modelo Declarativo/Procedimental de Ullman (2001c). No nível linguístico propomos a integração entre os achados neurais e as postulações procedimentais tratadas nos níveis anteriores e, elaboramos uma hipótese acerca da conexão entre a presença de possíveis disfunções computacionais nas regras gramaticais e estruturação sintática, ou seja, na memória procedimental e o processamento linguístico das PQG.

Para fundamentar experimentalmente essa investigação aplicamos o teste de memória procedimental ou aprendizagem implícita, ASRT - Alternating Serial Reaction Time (Tempo de Reação Serial Alternada) adaptado de Jesus (2012), com o intuito de avaliar a memória procedimental das PQG e compará-la a dos FF.

\section{MODELO PRÉ-MOTOR DUPLO}

O Modelo Pré-Motor Duplo, proposto por Alm (2005), surgiu com o objetivo de agregar os diversos achados experimentais encontrados até o momento sobre a gagueira e correlacioná-los com as explicações teóricas já existentes. Ou seja, segundo Alm, procurou-se dar sentido às "peças" espalhadas do "quebracabeças" da gagueira. Assim, em 2005, Alm concluiu a sua tese sobre os mecanismos causais desse transtorno da fluência e desenvolveu a teoria da gagueira baseada nos sistemas pré-motores duplos.

Segundo Alm (2004), a associação da gagueira à presença de disfunções nos núcleos da base ${ }^{2}$, não é recente. $\mathrm{Na}$ verdade, outros autores como Caruso (1991) e Molt (1999), considerados os precursores dessa perspectiva teórica, já iniciaram as pesquisas sobre os processos neuromotores subjacentes à gagueira e o possível papel dos núcleos da base nesse distúrbio da fluência.

De acordo com Alm (2005), existem vários indícios de que os núcleos da base estão intimamente relacionados com a gagueira. O primeiro indício diz respeito ao efeito de agentes farmacológicos que pioram ou amenizam a gagueira, pois eles afetam diretamente a dopamina, principal neurotransmissor que regula essas estruturas. O segundo indício refere-se à influência emocional, uma vez que distúrbios nos núcleos da base agravam diante de "tensão nervosa" e melhoram sob condições de relaxamento, o que é algo bem característico em pessoas portadoras de gagueira. O terceiro indício está relacionado às lesões que causam a gagueira adquirida, visto que frequentemente, elas afetam os núcleos da base.

Baseado nestes indícios e na observação de algumas situações indutoras da fluência em PQG como, por exemplo, o canto, a leitura em coro, a imitação, a encenação, a fala acompanhando o ritmo de um metrônomo, somado aos diversos achados de neuroimagem, efeitos de agentes farmacológicos e os estudos de Goldberg (1985) e Goldberg e Bloom (1990) - sobre o sistema motor humano, Alm (2005) desenvolveu um modelo explicativo para a gagueira relacionando esse distúrbio a disfunções nos núcleos da base (figura 1) e, considerando que o cérebro apresenta, basicamente, dois sistemas pré-motores paralelos que são responsáveis pelo planejamento e execução de movimentos, dentre eles, a fala. Estes sistemas, chamados sistema pré-motor lateral e sistema pré-motor medial (figura 2), promovem sinais de disparo para a realização de movimentos, no entanto, em condições um pouco diferentes.

\footnotetext{
tipos de sistemas de processamento da informação, possibilidade essa que vai de encontro a uma necessidade das ciências cognitivas, que é de não esbarrar no problema da unificação. Conforme Corrêa (2008), no contexto da ciência cognitiva, esse problema pode ser entendido como uma possibilidade de se considerar uma descrição em um nível como equivalente a outra pertencente a outro nível. Dessa forma, a proposta de Marr veio contribuir com a amenização desse problema, quando estabeleceu os seguintes níveis de descrição para o estudo dos sistemas de processamento da informação: 1) Nível computacional: referente às operações computacionais por meio das quais uma tarefa é conduzida; 2) Nível representacional ou algorítmico: referente à quais representações e mecanismos são utilizados pelo sistema para processar determinadas informações; 3) Nivel implementacional: referente à quais estruturas físicas estão envolvidas no processamento e, como essas estruturas operacionalizam suas atividades. A partir dessa proposta de Marr (1982) é que esquematizamos os níveis de descrição do presente estudo, de modo que o nível neural do nosso estudo está para o nível implementacional de Marr, assim como o nível procedimental está para o nível representacional e o nível linguístico está para o nível computacional.

${ }^{2}$ Estruturas responsáveis pelo processo de automatização de diversas funções cognitivas, motivacionais e de controle motor (ALM, 2005).
} 
Figura 1 - Núcleos da base e tálamo.

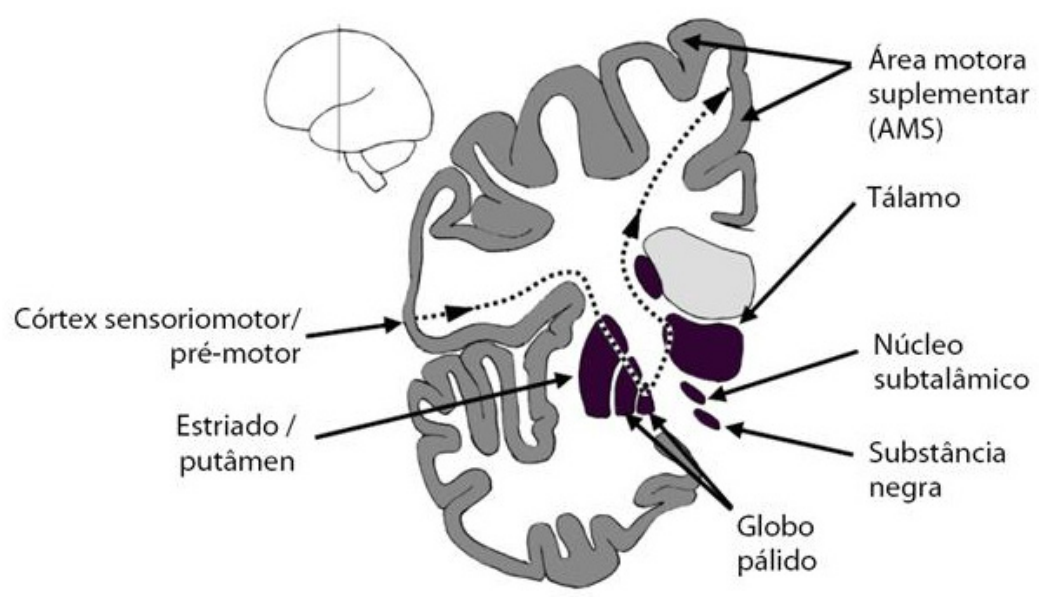

Nota: O circuito motor dos núcleos da base está t marcado esquematicamente pela linha pontilhada, do córtex motor até a área motora suplementar (AMS), passando pelo putâmen, globo pálido externo, globo pálido interno e tálamo. Fonte: Alm (2006).

Figura 2 - Os sistemas pré-motores duplos

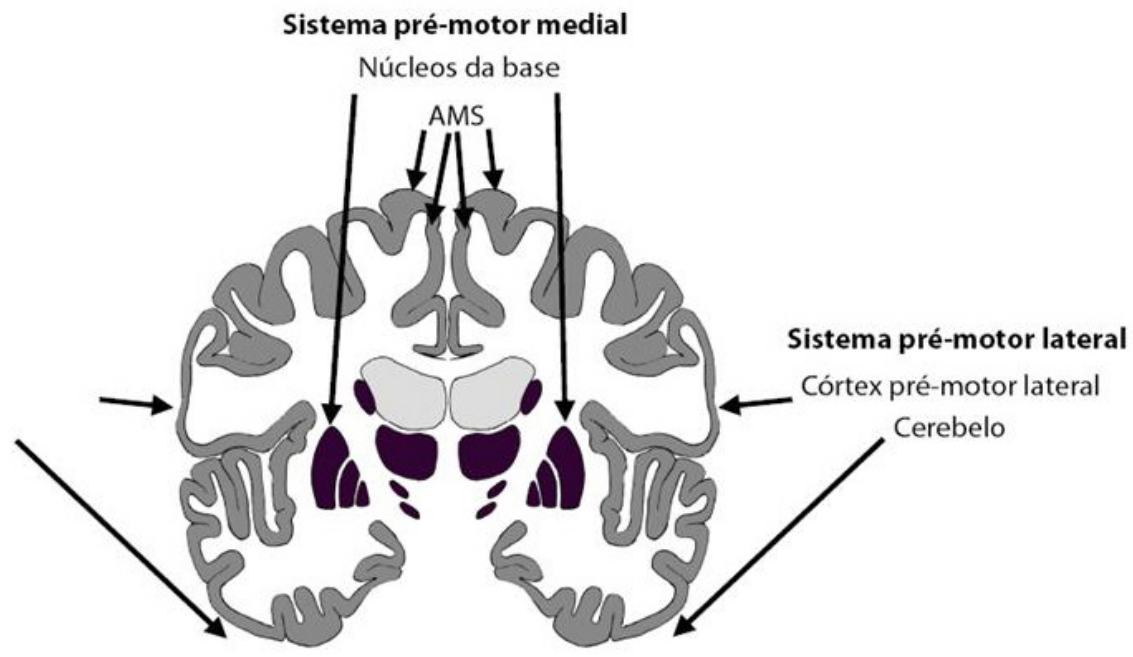

Nota: Baseado em Goldberg (1985, 1990) e Passingham (1987). Fonte: Alm (2006).

O sistema pré-motor lateral é formado pelo córtex pré-motor lateral e pelo cerebelo e encontra-se ativo quando o controle do movimento está sob a influência de um input sensorial, como quando se lê em coro, se fala acompanhando o ritmo de um metrônomo, ou ainda, quando se controla a fala por meio de feedback auditivo. Sobre este sistema, Alm afirma que ele também possui a capacidade de controlar a temporalização e sincronização da fala mesmo quando o input externo não é explícito, mas exige uma atenção aumentada tornando a fala menos automática e mais consciente, como é o caso da imitação, da encenação e do canto. Já o sistema pré-motor medial, por sua vez, é formado pelos núcleos da base e pela área motora suplementar e atua com base em programas automatizados, sem feedback externo, sendo este, o sistema dominante durante a fala espontânea.

Alm (2005), por meio desse novo referencial teórico para entender a gagueira, procurou enfatizar o papel dos núcleos da base na tarefa de automatização e temporalização de sequências motoras rápidas utilizadas durante a fala e, da área motora suplementar, como estrutura complementar nesta tarefa, responsável pela sincronização desses movimentos automáticos. 
Um aspecto interessante dessa teoria é que o sistema pré-motor medial compreende não apenas os núcleos da base, mas, a alça completa que parte do córtex (área motora suplementar), vai até os núcleos da base e o tálamo e retorna ao córtex. O que significa dizer que um funcionamento atípico dos núcleos da base pode ser o resultado de qualquer alteração estrutural e/ou fisiológica presente em qualquer ponto desse circuito. Isso inclui desde problemas no input do córtex em direção aos núcleos da base, a problemas no tálamo, na área motora suplementar e em todos os tratos fibrosos de substância branca que conectam essas estruturas.

Alm (2006) aponta que esse circuito é constituído por uma via direta e uma via indireta. Essas duas vias trabalham em cooperação, de maneira que a via indireta, para modular a atividade do córtex frontal, fornece uma inibição difusa da atividade cortical, e a via direta fornece uma ativação focal da ação desejada. As duas vias são dominadas por diferentes tipos de receptores de dopamina (D1 e D2), resultando, consequentemente, em efeitos diferenciados do neurotransmissor. Segundo Mink e Thach (1993) o fornecimento de pistas de temporalização para a área motora suplementar é uma tarefa dos núcleos da base e está vinculada a um bom desempenho da razão "sinal-ruído", ou seja, necessita de uma clara distinção entre a ativação focal realizada por uma via e a inibição difusa do córtex, realizada pela outra via. Uma ativação focal muito fraca da via direta resultaria em uma ativação aquém da desejada, levando a dificuldades na inicialização dos movimentos da fala, por exemplo. E também a presença de problemas na inibição difusa do córtex, realizada pela via indireta, poderia resultar na liberação de movimentos involuntários e em dificuldades de liberação dos movimentos voluntários, como observados na fala das PQG durante a ocorrência dos bloqueios, por exemplo.

Outro aspecto tratado por esse modelo refere-se à explicação apresentada por Alm (2005) para o início precoce e a remissão da gagueira em crianças com faixa etária entre, 2 anos e meio e 3 anos de idade. Sobre este aspecto, Alm afirma que o surgimento da gagueira e a alta porcentagem de remissões espontâneas ocorridas nessa idade, estão associadas a uma fase natural de desenvolvimento dos circuitos que constituem os núcleos da base, onde as crianças nessa idade, de uma maneira geral, apresentam um pico no número de receptores dopaminérgicos do tipo D2 nos núcleos da base. Por essa razão, existem algumas postulações teóricas argumentando que, quanto maior o número de receptores $\mathrm{D} 2$, maior o risco da criança em desenvolver a gagueira. Alm (2006) refere ainda que um elevado número de receptores $\mathrm{D} 2$ promove uma redução da inibição geral do córtex, além disso, a baixa razão entre receptores D1/D2 poderia causar um prejuízo no output dos núcleos da base.

Sobre a prevalência de a gagueira ocorrer mais em meninos do que em meninas, Alm (2006) também sugere que a relação $\mathrm{D} 1 / \mathrm{D} 2$ tende a ser mais baixa em meninos do que em meninas na infância, possivelmente influenciando a prevalência de gênero na gagueira. De um modo geral, parece que o padrão típico de surgimento e de remissão da gagueira na infância está relacionado, em grande parte, a uma fase natural no desenvolvimento do sistema dopaminérgico nos núcleos da base.

Dessa forma, o modelo de Alm conclui que a gagueira está relacionada a um distúrbio no sistema pré-motor medial e que a sua teoria oferece subsídios esclarecedores para a maioria das condições indutoras de fluência em PQG. Para o autor, a gagueira ocorreria como resultado de vários fatores atuando sobre o sistema prémotor medial, que poderia ser desde uma deficiência no input originário das regiões do córtex motor para os núcleos da base, até uma baixa relação entre os receptores de dopamina em qualquer parte do sistema, por exemplo.

Diante de todo este apanhado teórico, o que podemos perceber é que o modelo proposto por Alm dá conta apenas de explicar a gagueira no seu nível neural, mas não podemos aplicá-lo, diretamente, à análise de questões de processamento linguístico e modelos de gramática. Dessa forma, partimos para a próxima seção, onde discutiremos o modelo de memória proposto por Ullman (2001c) que corresponde ao nível procedimental deste estudo.

\section{MODELO DECLARATIVO/PROCEDIMENTAL}

O modelo declarativo/procedimental proposto por Ullman (2001c) postula que a linguagem depende de duas capacidades mentais, sendo elas, o léxico mental (capacidade memorizada) e a gramática mental (capacidade computacional). Sobre o léxico mental, Ullman o considera como um repositório de informações armazenadas que inclui desde estruturas linguísticas complexas, como sentenças cujo significado não pode 
ser obtido de forma clara, como as expressões idiomáticas, por exemplo, até estruturas mais simples como as formas irregulares das palavras. No entanto, diferentemente do léxico mental, a gramática mental diz respeito ao conhecimento implícito das regras que subjazem as operações mentais envolvidas na manipulação das palavras e, nas representações abstratas necessárias para a construção de estruturas complexas.

Portanto, Ullman (2001c, 2004 e 2008) estabelece que a premissa básica do seu modelo consiste na proposta de que o léxico e a gramática estão ligados a dois sistemas de memória distintos, sendo eles, o sistema de memória declarativa e o sistema de memória procedimental, respectivamente. No entanto, Ullman não postula que esses dois sistemas de memória são exclusivos para a sustentação do léxico e da gramática, de modo que outros componentes cognitivos ou computacionais podem estar relacionados a ambos os sistemas.

Assim, o sistema de memória declarativa estaria relacionado com a aprendizagem, representação e utilização de conhecimentos semânticos e episódicos, sendo um tipo de memória associativa, responsável não apenas pelo armazenamento de fatos e acontecimentos, mas também pelo conhecimento lexical, que inclui os sons e os significados das palavras, além de não ser informacionalmente encapsulado, porém, acessível aos diversos sistemas mentais, possuindo como estruturas cerebrais subjacentes, o lobo temporal medial e o hipocampo. Já o sistema de memória procedimental estaria ligado ao aprendizado inconsciente do processamento motor, perceptual, das habilidades cognitivas e a utilização da gramática através dos domínios da sintaxe, morfologia e, possivelmente, da fonologia, na perspectiva de como os sons podem ser combinados sequencialmente. Esse sistema estaria enraizado em partes do córtex frontal que inclui a área de Broca e a área motora suplementar, nos núcleos da base, no córtex parietal e no núcleo denteado do cerebelo. Diferentemente do sistema de memória declarativa, o sistema de memória procedimental estaria informacionalmente encapsulado, tendo, relativamente, pouco acesso aos sistemas mentais.

Para facilitar a compreensão desses sistemas de memória que trata o modelo declarativo/procedimental, abaixo, apresentamos o quadro 1, extraído do estudo de Walenski e Ullman (2005) sobre a ciência da linguagem com as principais características de cada memória.

No que se refere à comparação entre o modelo de Ullman e as demais correntes teóricas que tratam a respeito das bases neurais da linguagem, Ullman (2001a,b,c) afirma que o seu modelo assemelha-se ao modelo de sistema dual por atribuir componentes cognitivos e neurais distintos, inatos e especializados à gramática e ao léxico (CHOMSKY, 1995; PINKER, 1994) e, difere-se do modelo de sistema único, pelo fato das teorias que o compõem, postularem que tanto as palavras quanto as regras gramaticais são aprendidas e utilizadas por um único sistema, não específico da linguagem e com ampla distribuição anatômica cerebral (ELMAN et al., 1996; BATES, MACWHINNEY, 1989).

Segundo Ullman (2001c), o que difere o modelo declarativo/procedimental dos demais modelos, está relacionado às previsões sobre o armazenamento e acesso das formas lexicais regulares e irregulares. Pois, no que concerne a generalidade do domínio, apenas o modelo declarativo/procedimental, segundo o autor, estabelece associações entre a aprendizagem, representação e processamento de formas irregulares, além da associação entre as formas regulares, aspectos de sintaxe, habilidades motoras e cognitivas. No que diz respeito ao aspecto referente à localização, Ullman afirma que o seu modelo estabelece ligações entre as capacidades de linguagem (léxico e gramática) e o conjunto de estruturas cerebrais envolvido com essas capacidades, no entanto, diferentemente dos sistemas duais, o modelo declarativo/procedimental especifica as características neuroanatômicas dessa ligação.

Em seu modelo, Ullman também procurou discorrer sobre algumas evidências que comprovam o funcionamento atípico dos sistemas de memória em pessoas portadoras de patologias que afetam diretamente as estruturas cerebrais envolvidas, tanto no enraizamento da memória declarativa, quanto da procedimental. Entretanto, nesta seção, daremos ênfase apenas aos distúrbios que afetam os núcleos da base e/ou a área motora suplementar, por serem as áreas afetadas em pessoas portadoras de gagueira.

Sobre a Síndrome de Tourette $^{3}$ (ST), Ullman (2008) afirmou, em um estudo sobre a investigação do processamento gramatical e lexical em crianças portadoras da ST, por meio de uma tarefa de produção de

\footnotetext{
${ }^{3}$ Distúrbio do movimento caracterizado pela presença de tiques vocais e/ou motores associados à movimentos involuntários. As pessoas portadoras desta síndrome apresentam alterações neurofisiológicas nos núcleos da base, caracterizadas por níveis anormais de dopamina (ULLMAN, 2008).
} 
pretérito de formas regulares e irregulares, em língua inglesa, que as crianças com Tourette eram significativamente mais rápidas do que as crianças do grupo controle quanto à produção de formas regulares no pretérito, mas, apresentaram desempenho semelhante às crianças sem Tourette, quanto à produção de pretéritos das formas irregulares. Assim, concluiu-se que o processamento de informações relacionadas à memória procedimental mostrou-se acelerado, consequentemente, atípico, sugerindo que as disfunções nos núcleos da base podem acarretar não só os tiques motores ou vocais, mas também provocar alterações na forma como essas pessoas processam informações gramaticais.

Quadro 1 - Visão geral das bases funcionais e biológicas dos sistemas de memória declarativa e procedimental

\begin{tabular}{|c|c|c|}
\hline & SISTEMAS DE MEMÓRIA & \\
\hline Especializada para a aprendizagem: & Memória Declarativa & Memória Procedimental \\
\hline Velocidade de aprendizagem & Rápido & Gradual \\
\hline $\begin{array}{l}\text { O conhecimento } \quad \text { aprendido } \\
\text { conscientemente acessível? }\end{array}$ & $\begin{array}{l}\text { Tipicamente, } \\
\text { necessariamente. }\end{array}$ & $N \tilde{a} o$ \\
\hline Funções não linguísticas & $\begin{array}{l}\text { Aprendizagem e utilização de } \\
\text { fatos e eventos }\end{array}$ & $\begin{array}{l}\text { Aprendizagem e utilização de } \\
\text { habilidades motoras } e \\
\text { cognitivas }\end{array}$ \\
\hline Funções linguísticas & $\begin{array}{l}\text { Aquisição, representação } \\
\text { processamento de conhecimento } \\
\text { lexical, incluindo formas } \\
\text { complexas memorizadas. }\end{array}$ & $\begin{array}{l}\text { Aquisiçãa, representação } e \\
\text { processamento de estruturas } \\
\text { complexas através da sintaxe, } \\
\text { morfologia e, possivelmente, } \\
\text { fonologia e semântica de } \\
\text { composição. }\end{array}$ \\
\hline Importantes estruturas cerebrais & & \\
\hline Subcortical/Medial: & Hipocampo & Núcleos da base \\
\hline Neocortical: & $\begin{array}{l}\text { Principalmente regiões do lobo } \\
\text { temporal }\end{array}$ & $\begin{array}{l}\text { Principalmente regiões do lobo } \\
\text { frontal, especialmente o córtex } \\
\text { pré-motor e a área posterior de } \\
\text { Broca. }\end{array}$ \\
\hline Hormônios e neurotransmissores & Estrogênio e acetilcolina & Dopamina \\
\hline
\end{tabular}

Fonte: Walenski e Ullman (2005).

Sobre a Doença de Parkinson, associada à degeneração dos neurônios dopaminérgicos, principalmente na substância negra dos núcleos da base, ocorre a diminuição da atividade motora, denominada hipocinesia, resultando em dificuldades na expressão de sequências motoras e, no processamento gramatical. A respeito desta última dificuldade, Ullman (2008) reporta a presença de mais erros gramaticais, por parte dos portadores de Parkinson, na produção de verbos regulares do que, na produção de verbos irregulares no pretérito. O que contribui para a postulação do modelo declarativo/procedimental, que propõe que as formas irregulares são armazenadas na memória declarativa e, a flexão de verbos regulares na memória procedimental, que, nessas pessoas encontra-se deficitária devido ao acometimento estrutural dos núcleos da base. 
Na doença de Huntington, assim como na de Parkinson, também ocorre a degeneração dos núcleos da base. Mas, ao contrário da doença de Parkinson identifica-se um quadro de hipercinesia (presença de movimentos excessivos) e uma tendência a super-sufixação de verbos, o que leva a acreditar, de acordo com o modelo de Ullman, que estas estruturas cerebrais estão subjacentes à expressão tanto das regras gramaticais quanto do controle motor, indicando a existência de funções similares nos dois domínios.

Dessa forma, pode-se afirmar que a análise por planos complementares, na forma como feita neste capítulo e assumida nessa dissertação, mostra-se adequada para uma caracterização mais ampla das questões concernentes às relações entre cérebro e linguagem. Nesse modo de abordagem, respeitam-se as propriedades de cada nível, sem a necessidade de se operar uma redução que, forçosamente, leva a problemas de ordem ontológica, na medida em que os objetos descritos em cada nível, no caso, neurológico, funcional e linguístico, não podem ser ajustados às unidades dos outros níveis, visto que isso traria problemas de incomensurabilidade (FERRARI-NETO, 2012), além de levantar questões relativas às diferenças de granularidade entre os construtos teóricos elaborados nos respectivos campos de conhecimento (Neurociências, Psicolinguística e Linguística) dedicados a cada nível, o que jaz no campo dos problemas epistemológicos (FERRARI-NETO, 2012). Assim, uma abordagem em planos complementares supera esses obstáculos, caracterizando os fenômenos em cada nível, em separado, porém estabelecendo as devidas correlações entre eles, garantindo uma explicação global destes fenômenos. Tal modelo de análise será aplicado aos dados empíricos colhidos a partir dos experimentos conduzidos no âmbito deste trabalho, os quais passam a ser descritos a seguir.

\section{HIPÓTESE DE TRABALHO}

A hipótese de trabalho deste artigo baseia-se no desenvolvimento articulado entre os níveis neural e procedimental, através do qual se tornou possível perceber que existem alguns pontos em comum entre o Modelo Pré-Motor Duplo de Alm (2005), que representa o nível neural, e o Modelo Declarativo/Procedimental de Ullman (2001c), relativo ao nível procedimental. Estas semelhanças estão fundamentadas basicamente em dois aspectos.

O primeiro aspecto trata-se das estruturas cerebrais nas quais estão enraizados o sistema pré-motor medial (que se apresenta com alterações nas PQG) e o sistema de memória procedimental. Ambos os sistemas compartilham os núcleos da base e a área motora suplementar como estruturas cerebrais que subjazem o seu funcionamento, e que estão intimamente relacionadas à automatização de diversas funções cognitivas e do controle motor. O segundo aspecto diz respeito às evidências experimentais que apontam para um funcionamento atípico da gramática ${ }^{4}$ em pessoas que apresentam alterações neurofisiológicas nos núcleos da base e na área motora suplementar, a exemplo das pessoas portadoras da Doença de Parkinson, da Doença de Huntington e da Síndrome de Tourrett, conforme Ullman (2008). Desse modo, concebemos que, semelhantemente a essas desordens referidas, as pessoas que gaguejam também estariam mais suscetíveis a um funcionamento atípico da gramática.

Partindo desse pressuposto, propõe-se a hipótese de que a presença de alterações neurofisiológicas no sistema pré-motor medial em PQG, que constituem uma das causas deste distúrbio, seriam também responsáveis por um funcionamento atípico de sua gramática. Assim como os demais portadores de patologias, que apresentam acometimento nos núcleos da base e na área motora suplementar evidenciam alterações no processamento da gramática, as PQG também sofreriam a influência deste comprometimento na memória procedimental, tendo em vista o Modelo Declarativo/Procedimental de Ullman. Dessa forma, ficam articulados nessa hipótese, os três níveis de descrição sugeridos por Marr (1982).

\footnotetext{
${ }^{4}$ Por gramática, assumimos aqui a ideia de que este termo se refere ao conhecimento linguístico internalizado que um falante possui sobre a sua língua; nesse sentido, aproxima-se da noção de língua-I, conforme proposto por Chomsky (1995). Tal conhecimento seria composto por um sistema computacional, definido como um conjunto de operações linguísticas que explicitam os passos derivacionais necessários para a geração de sentenças nessa língua, e por um léxico, que armazenaria as unidades atômicas sobre as quais as operações do sistema computacional agiriam, na geração de sentenças. Ainda que Ullman, em seus trabalhos, não deixe claro que componente da gramática está sendo referido pelo termo gramática mental, entendemos aqui que ele concerne apenas ao sistema computacional, que estaria enraizado na memória procedimental, excluindo o léxico, que estaria baseado na memória declarativa.
} 


\section{METODOLOGIA}

O presente estudo se caracteriza como uma pesquisa experimental, exploratória, comparativa e quantitativa, uma vez que fizemos uso de algumas técnicas da Psicolinguística Experimental para a investigação da hipótese lançada e a partir de uma análise estatística comparativa entre grupos, obtivemos os resultados e as conclusões da pesquisa. A certidão de aprovação do estudo foi emitida em 23 de abril de 2013, pelo Comitê de Ética em Pesquisa do Centro de Ciências da Saúde da Universidade Federal da Paraíba UFPB/CEP/CCS (Anexo 01), com base na Resolução 196/96 que regulamenta a pesquisa envolvendo seres humanos.

As PQG participantes do estudo compuseram o grupo experimental e os $\mathrm{FF}$ fizeram parte do grupo controle. Alguns voluntários do grupo experimental eram pacientes da Clínica-Escola de Fonoaudiologia da UFPB, de clínica fonoaudiológica particular e membros do Grupo de Orientação Discutindo-Gagueira do Instituto Brasileiro de Fluência em João Pessoa/PB. Todos esses participantes não apresentaram história de lesões neurológicas e a realização de acompanhamento terapêutico fonoaudiológico, anterior ou durante a pesquisa, não foi utilizada como critério de exclusão do grupo.

Com o intuito de caracterizar as PQG quanto ao tipo e à severidade da gagueira, todos os voluntários do grupo experimental se submeteram a uma avaliação fonoaudiológica, realizada por meio do Protocolo do Perfil da Fluência da Fala do ABFW de Andrade (2004) (Anexo 02) e do Instrumento de Severidade da Gagueira - Stuttering Severity Instrument de Riley (1994), adaptado para o PB por Oliveira (2012). Como todos os participantes desse grupo eram pessoas com a gagueira do desenvolvimento persistente, buscamos caracterizá-los de acordo com o score de severidade, que apresentaremos na tabela 1 do próximo capítulo.

A aplicação da tarefa experimental se deu na sala do Laboratório de Processamento Linguístico da UFPB (LAPROL). Cada participante foi testado individualmente após a leitura e assinatura do Termo de Consentimento Livre e Esclarecido e, após receber as orientações pela experimentadora, lê-las na tela do computador e serem submetidos a uma sessão de prática, para familiarização com a tarefa.

\subsection{Teste de Tempo de Reação Serial Alternada}

A memória procedimental, conforme Lent (2010) é um tipo de memória de aprendizagem implícita de hábitos, habilidades e regras que necessita da repetição para ser armazenada. Devido a sua natureza implícita, Lent aponta que inúmeros neuropsicólogos encontraram, inicialmente, dificuldades para elaborar testes que eliminassem o conhecimento explícito da aprendizagem de procedimentos, isolando assim, a sua natureza implícita. Até que surgiu o teste $S R T$ - Serial Reaction Time (Tempo de Reação Serial), tarefa onde o pesquisador realiza a aferição do tempo médio que o participante leva para mover o dedo em resposta ao estímulo luminoso de comando, também chamado de tempo de reação. Nesse teste, solicita-se que o participante realize movimentos com os dedos da mão seguindo uma sequência que lhe parece aleatória, mas que de fato, possui uma regularidade não percebida pelo participante. Através da medição do tempo de reação durante a realização do teste, é possível constatar que o desempenho melhora ao longo do tempo, o que significa que o participante aprendeu, implicitamente, a sequência de movimentos à medida que realizou uma série de repetições, como se pode observar na figura 3.

A partir desse contexto clássico de testes de memória procedimental, como o SRT, a tarefa ASRT Alternating Serial Reaction Time (Tempo de Reação Serial Alternada) foi desenvolvida a partir da necessidade de tornar a regularidade das sequências motoras ainda mais imperceptível, desse modo, como aponta Nemeth et al. (2011), no teste $A S R T$, a repetição de eventos é alternada com elementos aleatórios. Isto significa dizer que a localização de cada segundo estímulo na tela do computador é determinada aleatoriamente, por exemplo, na sequência 4512, em que os números representam os seus locais respectivos na tela do computador, esta sequência de estímulos possuirá um elemento aleatório entre eles, apresentando-se da seguinte maneira: $4 \mathrm{R} 5 \mathrm{R} 1 \mathrm{R} 2 \mathrm{R}$, com "R" representando um elemento aleatório. Por ser a tarefa mais utilizada atualmente para a avaliação desse tipo de memória, nesta dissertação, fizemos uso do $A S R T$ para investigar a memória procedimental dos participantes do grupo experimental e do grupo controle. 
Figura 3

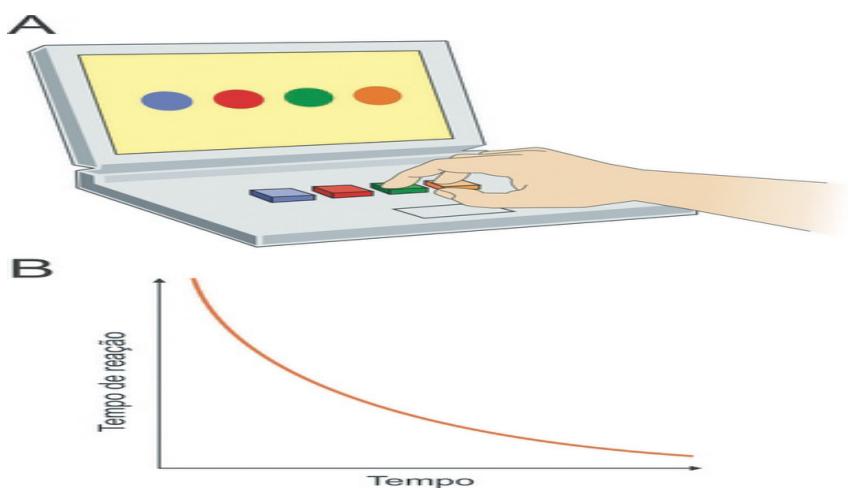

Nota: Teste SRT com a utilização de cores como estímulo visual (A). Gráfico evidenciando a diminuição do tempo de reação do participante ao longo a tarefa (B). Fonte: Lent (2010).

\section{EXPERIMENTO}

Com o intuito de investigar a memória procedimental dos participantes da pesquisa, realizamos o teste ASRT com o grupo experimental, formado pelas PQG e com o grupo controle, formado pelos FF, com o objetivo de estabelecer uma análise comparativa no que concerne ao grau de aprendizagem implícita de ambos os grupos.

\subsection{Método}

Participantes:

Participaram voluntariamente deste experimento $9 \mathrm{FF}$, sendo 8 do sexo feminino e 1 do sexo masculino com idade média de 22 anos e, 8 pessoas com a gagueira do desenvolvimento persistente (Persistent Developmental Stuttering - PDS), sendo 2 do sexo feminino e 6 do sexo masculino com idade média de 28 anos. Abaixo apresentamos a tabela 1, com a respectiva distribuição das PQG que compuseram o grupo experimental.

Tabela 1 - Distribuição dos participantes do grupo experimental quanto à severidade da gagueira

\begin{tabular}{lll}
\hline Participante & Score SSI & Severidade \\
\hline 1 & 15 & Muito Leve para Leve \\
2 & 17 & Muito Leve para Leve \\
3 & 18 & Leve \\
4 & 19 & Leve \\
5 & 27 & Moderada \\
6 & 30 & Moderada para Grave \\
7 & 38 & Muito Grave \\
\hline 8 & 52 & Muito Grave \\
\hline
\end{tabular}

Fonte: Dados do SSI. 
Como observamos na tabela, de um modo geral, a severidade da gagueira apresentou-se distribuída na amostra de maneira equilibrada, com basicamente 2 participantes para cada nível de severidade, exceto para a severidade moderada e moderada para grave.

Material:

Adaptamos o teste ASRT utilizado na dissertação de Daniela Brito de Jesus (2012) e programamos na versão 2.0 do Paradigm, produzido pela empresa Paradigm Experiments disponível no site www.paradigmexperiments.com e instalado em um Notebook SAMSUNG, modelo NP300E4A com as teclas $1,2,4$ e 5 devidamente etiquetadas e sinalizadas para a realização da tarefa, cada uma correspondendo a um dos círculos da tela, da esquerda para a direita, como demonstra a figura 4. Esta tarefa era formada por 1 ciclo com 85 estímulos aleatórios destinado à sessão de prática dos participantes e, por 15 ciclos experimentais, com 11 posições-alvo (sequência numérica 4512), totalizando 85 estímulos por ciclo.

Figura 4- Teclas numéricas correspondentes às posições do smile na tela

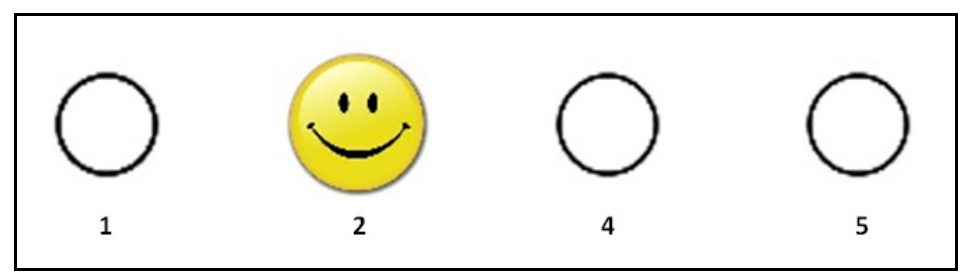

Nota: Variável Independente: Posição-alvo (sequência numérica 4512). Variável Dependente: Tempo médio de reação à posição-alvo em milissegundos $(m s)$.

\subsection{Procedimento}

Para a realização da tarefa "Pegue o Smile", todos os participantes foram testados individualmente e receberam as orientações pela experimentadora e através da tela do computador (figura 5). Como estímulo visual, apresentamos um smile que aparecia em um dos quatro círculos vazios na tela do computador e os sujeitos foram orientados a pressionar a tecla correspondente à sua localização espacial (figura 6).

Figura 5 - Tela inicial do teste ASRT

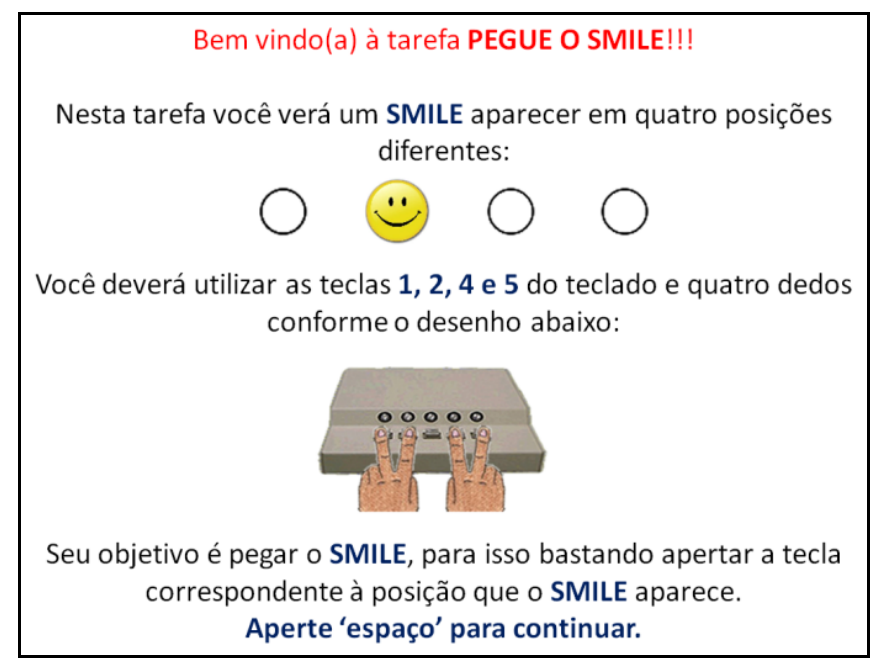

Ao apertar a tecla numérica correspondente à posição do smile na tela, o estímulo visual desaparecia e, automaticamente, apresentava-se o próximo estímulo. Nesta tarefa, o próprio participante era responsável pelo controle do tempo de visualização de cada estímulo, registrado pelo computador em milissegundos $(m s)$. 
Figura 6 - Sequência de telas iniciais da prática no teste ASRT

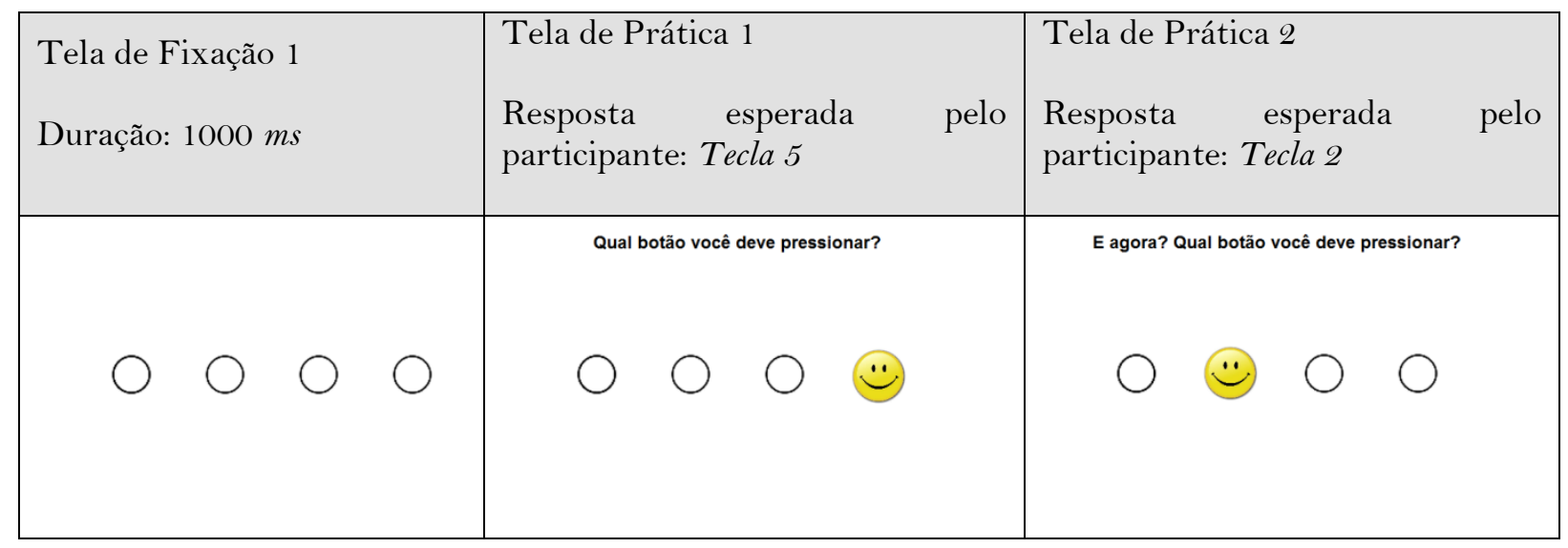

Logo após os 8 primeiros blocos (figura 7), inserimos um intervalo de quinze segundos para que o participante pudesse descansar um pouco e retomar a tarefa. Ao término dos quinze segundos, apresentamos uma nova tela com um lembrete acerca do posicionamento dos dedos para a realização da tarefa e solicitando que o participante apertasse a tecla "espaço" para continuar. Desse modo, os participantes realizaram mais uma sequência de 7 ciclos, totalizando os 15 ciclos experimentais. Completado todos os ciclos, os participantes visualizavam uma nova tela informando que a tarefa havia chegado ao fim e agradecendo a participação.

Figura 7 - Desenho esquemático do experimento

\begin{tabular}{|l|l|l|l|l|l|l|l|l|l|l|l|l|l|l|l|l|l|}
\hline Prática & Intervalo & 1 & 2 & 3 & 4 & 5 & 6 & 7 & 8 & Intervalo & 9 & 10 & 11 & 12 & 13 & 14 & 15 \\
\hline
\end{tabular}

\subsection{Hipótese e Previsões}

Se a gagueira está relacionada a um distúrbio no sistema pré-motor medial e as estruturas que o compõem são as mesmas que enraízam a memória procedimental, então, podemos supor que as pessoas que gaguejam estarão sujeitas à influência deste comprometimento e apresentarão dificuldades na tarefa de aprendizagem implícita, ou seja, na memória procedimental, em relação aos FF. Esta dificuldade pode ser evidenciada como uma manutenção ou aumento do tempo de reação à posição-alvo, medido em milissegundos. Ao contrário do que se espera para os FF, ou seja, espera-se que estes apresentem uma redução do tempo de reação ao longo da tarefa, demonstrando que aprendeu a sequência implicitamente, tornando-a cada vez mais automática.

\subsection{Resultados e Discussão}

Os gráficos 1 e 2, abaixo, apresentam os resultados encontrados.

Uma análise visual do gráfico 1 aponta para uma tendência dos FF em reduzir o tempo de reação com o aumento da quantidade de ciclos, ao contrário da tendência apresentada pelas PQG no gráfico 2, que mostra-se invertida, ou seja, a medida que a quantidade de ciclos aumenta, também aumenta o tempo de reação à posição-alvo. Uma análise de correlação de Spearman mostrou um coeficiente positivo para a variável ciclo $(\pi=0.05)$, indicando que as PQG vão ficando ligeiramente mais lentas conforme o aumento do número de ciclos. Uma análise do grau de correlação entre as variáveis controladas neste experimento mostra que elas estão correlacionadas em alta medida $(p=0.78)$, assumindo-se que o grau de correlação é considerado alto quando acima de .60, o que indica que as variáveis se inter-relacionam a um grau de $60 \%$. Para os FF o mesmo padrão se verificou, apenas alterando-se o sinal do coeficiente de correlação, ainda para a variável ciclo, que desta vez se relevou negativo $(\pi=-0.06)$, indicando que os sujeitos desse grupo ficam ligeiramente mais rápidos conforme aumenta o número de ciclos. Mais uma vez, o grau de correlação ficou também no nível considerado alto $(\mathrm{p}=0.74)$, repetindo-se o que foi observado para o grupo experimental. Ainda assim, observou-se um efeito relativamente pequeno, dada a sutileza do fenômeno, desse modo, 
precisaríamos de um número maior de ciclos ou de participantes, para que essa diferença atingisse uma magnitude maior, que se refletiria no tamanho do efeito estatisticamente encontrado pelo modelo.

Gráfico 1 - Tempo médio de reação dos FF no teste ASRT em milissegundos (ms)

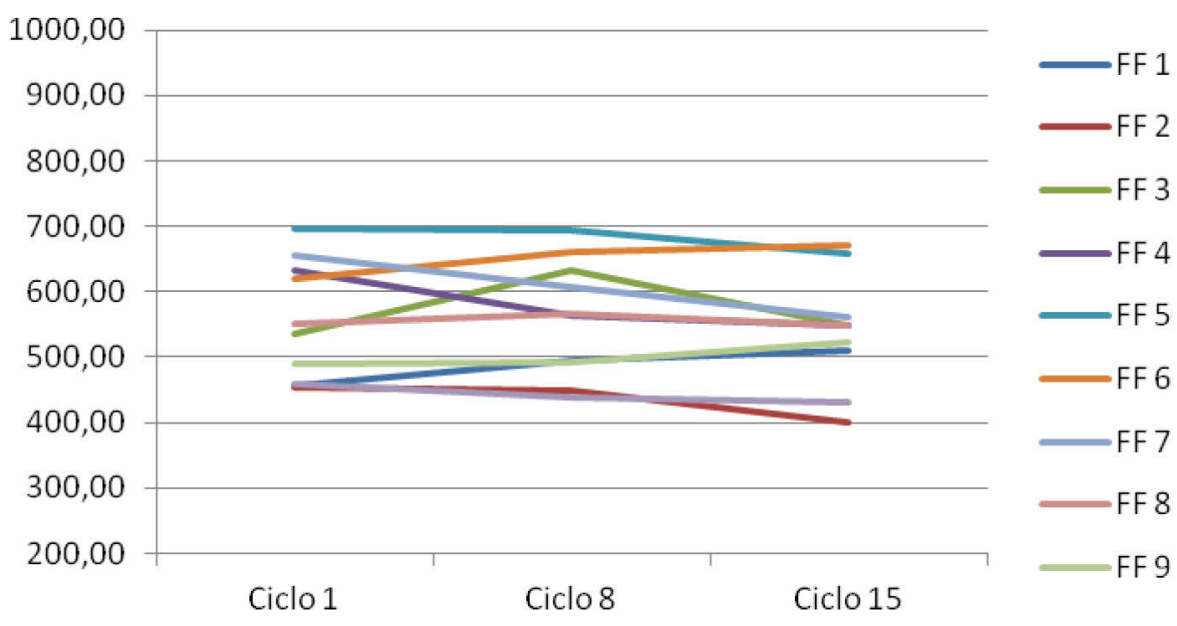

Gráfico 2 - Tempo médio de reação das PQG no teste ASRT em milissegundos (ms)

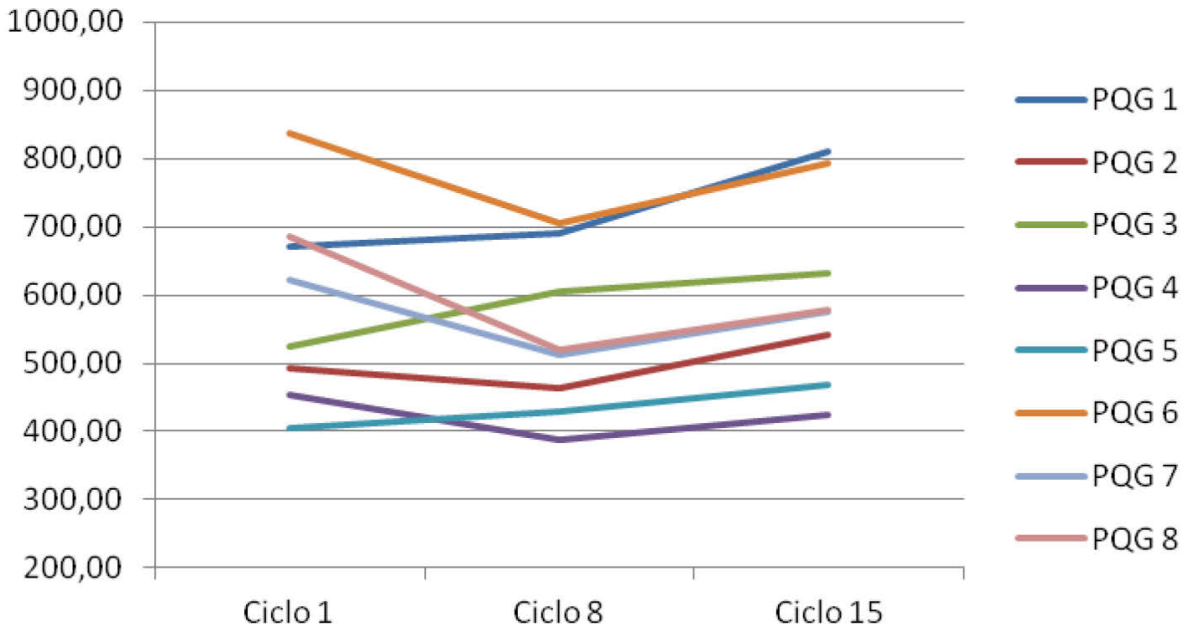

A fim de investigar a presença de uma possível associação entre o nível de severidade da gagueira, obtido por meio do SSI, e o desempenho das PQG no teste ASRT, submetemos os dados a uma matriz de correlação de Spearman e não constatamos efeito de interação entre o score do SSI e a média do tempo de reação das PQG na tarefa ASRT $(\pi=0.5 \mathrm{e} \mathrm{p}=0.21)$, sugerindo, portanto, que o grau de severidade da gagueira não exerce influência sobre o seu desempenho no teste de memória procedimental. Possivelmente, esse efeito não tenha se tornado evidente pelo número reduzido de participantes, de modo que, caso tivéssemos mais participantes para cada grau de severidade, provavelmente, poderíamos apontar ou refutar esse efeito de interação com maior propriedade.

Dessa forma interpretamos o aumento do tempo de reação das PQG como uma dificuldade na aprendizagem procedimental das sequências numéricas na tarefa ASRT, consequentemente, na memória procedimental. Esses achados, ao passo que reforçam a teoria da gagueira baseada nos sistemas pré-motores duplos (ALM, 2005), por meio das evidências experimentais de que as PQG apresentam dificuldades na aprendizagem de sequências motoras, fornecem indícios de que essas dificuldades influenciam no funcionamento do sistema de memória procedimental, já que, ambos os sistemas, compartilham as mesmas estruturas neurais.

Com base nas evidências encontradas na literatura, a aprendizagem procedimental está diretamente relacionada com as habilidades motoras e cognitivas, principalmente quando nos referimos à fala, no que concerne às sequências de movimentos articulatórios e, à linguagem, em termos de processamento das 
regras gramaticais. Por essa razão, alguns pesquisadores resolveram estudar a linguagem e sua relação com a memória procedimental em pessoas que possuíam algum tipo de alteração neurofisiológica nos núcleos da base, estruturas neurais que enraízam o sistema de memória procedimental.

Assim, encontramos os estudos de Ullman, Corkin, Coppola, et al. (1997) e Ullman (2001c, 2004 e 2008) que realizaram experimentos com pessoas portadoras da Doença de Parkinson e de Huntington, e encontraram evidências de que a presença de dificuldades na memória procedimental influencia na maneira como essas pessoas produzem verbos regulares no passado, em língua inglesa. De modo semelhante, Ullman e Pierpont (2005) e Lum, Conti-Ramsden, Page e Ullman (2012) realizaram um estudo investigando o mesmo fenômeno linguístico, no entanto, com crianças portadoras do Distúrbio Específico de Linguagem (DEL) e também encontraram relação entre as dificuldades de linguagem evidenciadas pelas crianças e a presença de déficits na memória procedimental. Riascos e Castellanos (2011), baseados em um levantamento bibliográfico, também propuseram uma relação entre o distúrbio nos núcleos da base e a interferência dele na linguagem de pessoas com a Síndrome de Tourette.

No que diz respeito à relação entre memória procedimental e linguagem em pessoas que gaguejam, encontramos o estudo de Bauman (2009) que investigou o mesmo fenômeno das flexões de verbos regulares e irregulares no passado, entretanto, com crianças que gaguejam e crianças fluentes, falantes do inglês. Como resultado, a autora evidenciou uma tendência das crianças que gaguejam, em relação às FF, utilizarem mais as formas irregulares dos verbos no passado, do que as formas regulares. Sobre esse achado, a autora inferiu que as crianças que gaguejam apresentariam dificuldades na memória procedimental, que, por sua vez, seria responsável pelas regras gramaticais de flexão dos verbos regulares. Por essa razão, as crianças com gagueira fariam mais uso das formas irregulares, já que estariam armazenadas na memória declarativa e não seriam submetidas às regras gramaticais, processadas com o auxílio da memória procedimental. Richels, Buhr, Conture e Ntourou (2010) também em um estudo com crianças que gaguejam, estudaram a complexidade da elocução e a presença da gagueira em palavras de função e concluíram que, a investigação a respeito de como as palavras de função e as palavras de conteúdo são armazenadas e acessadas, podem ajudar a explicar por que as palavras de função são mais suscetíveis à gagueira do que as palavras de conteúdo, em crianças pré-escolares. Os autores reportaram ainda, a possibilidade das palavras de função e de conteúdo serem enraizadas em diferentes sistemas neurais, como aponta Ullman (2001c, 2004), que propôs a ligação das palavras de função à memória procedimental e das palavras de conteúdo à memória declarativa.

Em consonância com os nossos achados, Smits-Bandstra e De Nil (2007) reportam quatro estudos que partiram da previsão de que as PQG apresentam dificuldades de aprendizagem procedimental e de automatização de sequências, sustentadas pela hipótese da presença de disfunção no circuito básico dos núcleos da base. Para investigar essa hipótese, os autores resolveram estudar a habilidade das PQG em aprender sequências após um período de prática. Nos quatro experimentos reportados no estudo, as PQG foram significativamente mais lentas, em termos de tempo de reação, do que os $\mathrm{FF}$. O que emerge como uma sustentação de que, este comprometimento no circuito dos núcleos da base pode ser um componente etiológico no desenvolvimento e na manutenção da gagueira. Knopman e Nissen (1991) investigaram a aprendizagem procedimental em pessoas com a Doença de Huntington (DH), por meio do teste de reação serial (SRT), e observaram um aumento da latência de resposta à medida que se aumentava a quantidade de ciclos, o que caracterizou, segundo os autores, um déficit de aprendizagem procedimental.

Através desses estudos, observamos que existem algumas evidências experimentais que apontam para a relação entre a presença de alterações neurofisiológicas nas estruturas neurais que enraízam a memória procedimental e a presença de um comportamento atípico na linguagem dos seus portadores, para os fenômenos linguísticos investigados, o que robustece a nossa hipótese de que a presença de alterações neurais no sistema pré-motor medial em PQG, seriam também responsáveis por um funcionamento atípico de sua gramática, que investigaremos linguisticamente, em um próximo experimento, por meio do fenômeno da correferência anafórica ${ }^{5}$ intrassentencial.

Diante dos resultados que obtivemos neste experimento de memória procedimental, apesar das dificuldades de averiguação dos achados devido à própria natureza do teste, alcançamos o objetivo que consistia em

\footnotetext{
${ }^{5}$ Anáforas são expressões que não podem ter referência independente e por isso devem tomar a referência de um antecedente apropriado, inter ou intrassentencialmente (SILVA; BRITO, 2012).
} 
avaliar o comportamento desse tipo de memória tanto em PQG como em FF, além de corroborarmos com os vários estudos que investigam a relação entre distúrbios dos núcleos da base, aprendizagem procedimental e linguagem.

\section{CONSIDERAÇÕES FINAIS}

A perspectiva de análise por planos complementares nos auxiliou a não esbarrar no problema da unificação e possibilitou que articulássemos os achados neurais e procedimentais existentes sobre gagueira e memória e, a partir dessa relação, permitiu que postulássemos a hipótese de que a presença de alterações neurofisiológicas no sistema pré-motor medial em PQG, podem ser também responsáveis por um funcionamento atípico de sua gramática. Pois os resultados encontrados no teste ASRT apontam para uma tendência dos grupos em comportarem-se de maneira distinta, como o previsto.

Como a nossa hipótese fundamentou-se em evidências experimentais que apontam para uma maior suscetibilidade de funcionamento atípico da gramática em pessoas que apresentam disfunções neurofisiológicas nos núcleos da base e na área motora suplementar (estruturas também responsáveis por enraizar o sistema de memória procedimental), podemos dizer que os resultados obtidos oferecem indícios de que as PQG apresentam dificuldades na memória procedimental, o que corrobora com o direcionamento do nosso estudo à rejeição da hipótese nula. Acreditamos que mediante uma reformulação do ASRT para o aumento do número de estímulos, juntamente com um aumento significativo do número de participantes, podemos alcançar um efeito mais robusto.

O experimento que tratamos neste artigo consiste em uma etapa inicial e necessária, para identificarmos em que direção caminha a nossa hipótese de trabalho e desenvolvermos a investigação com base na perspectiva de análise por planos complementares. O próximo passo, portanto, consistirá no desenvolvimento do nível linguístico do estudo, aonde pretendemos investigar o funcionamento da gramática das PQG por meio do fenômeno da correferência intrassentencial, que, diferentemente da intersentencial, depende da atuação dos princípios de ligação considerados como estritamente linguísticos, ou seja, os princípios $\mathrm{A}^{6}, \mathrm{~B}^{7}$ e $\mathrm{C}^{8}$ (CHOMSKY, 1986) não parecem ser aplicados a nenhum outro domínio cognitivo e nem a princípios residentes em outros domínios que sejam correlatos a eles. Portanto, se esses princípios são estritamente gramaticais, linguísticos e estão radicados na memória procedimental e essa memória está enraizada nas estruturas cerebrais que apresentam alterações neurais nas PQG, podemos esperar que estas pessoas evidenciem um desempenho atípico no processamento dessa forma de correferência, já que supomos estarem esses princípios de ligação correlacionados com a gramática e a gramática com a memória procedimental. Nessa perspectiva é que estabeleceremos a relação entre os desempenhos obtidos pelos participantes no teste de memória procedimental, com os resultados linguísticos obtidos na tarefa de correferência e verificaremos se, como supomos, o processamento correferencial das PQG estará sujeito à influência de um possível déficit na memória procedimental.

\section{REFERÊNCIAS}

ALM, P. Stuttering and the basal ganglia circuits. Journal of communication disorders, v. 37, p. 325-369, 2004.

On the causal mechanisms of stuttering. 2005. 78fls. Thesis (Doctorate in Neuroscience) - Lund

University, Sweden, 2005.

. Um novo referencial para compreender a gagueira: o modelo pré-motor duplo. IFA Congress 2006; tradução: Hugo Silva; revisão técnica: Sandra Merlo. Disponível em:

http://www.gagueira.org.br/conteudo.asp?id_conteudo=133. Acesso em 17 de abril de 2012.

\footnotetext{
${ }^{6}$ Princípio A - uma anáfora deve ser presa no seu domínio de vinculação.

${ }^{7}$ Princípio B - um pronome deve ser livre no seu domínio de vinculação.

${ }^{8}$ Princípio C - uma expressão R(eferencial) deve ser livre em qualquer domínio.
} 
ANDRADE, C. R. F. Fluência. In: ANDRADE, C. R. F.; BEFI-LOPES, D. M.; FERNANDES, F. D. M.; WERTZNER, H. F. ABFW: Teste de linguagem infantil nas áreas de fonologia, vocabulário, fluência e pragmática. 2 ed. Barueri, SP: Pró-Fono, 2004.

BATES, E. A.; MACWHINNEY, B. Functionalism and the competition model. In: MACWHINNEY, B.; BATES, E. The crosslinguistic study of sentence processing. Cambridge University Press, 1989.

BAUMAN, J. The development of syntactic complexity and the irregular past tense in children who do and do not stutter. University of Maryland. Thesis, 2009.

CARUSO, A. J. Neuromotor processes underlying stuttering. In: PETERS et al. Speech motor control and stuttering, p. 101-116, 1991.

CHOMSKY, N. Knowledge of language, its nature, origin and use. New York: Praeger, 1986.

The minimalist program. Massachussetts, MIT Press, 1995.

CORRÊA, L. M. S. Relação processador linguístico-gramática em perspectiva: problemas de unificação em contex to minimalista. D.E.L.T.A., v. 24, p. 231-282, 2008.

ELMAN, J. L.; BATES, E. A.; JOHNSON, M. H, KARMILOFF-SMITH A.; PARISI D.; et al. Rethinking innateness: A connectionist perspective on development. Cambridge, Massachusetts: The MIT Press, 1996.

FERRARI-NETO, J. Ciências da linguagem e filosofia: uma análise das relações entre linguística, psicolinguística e neurociências sob a ótica da filosofia da mente. Letras de hoje, Porto Alegre, v. 47, p. 93101, 2012

GOLDBERG, G. Supplementary motor area structure and function: Review and hypotheses. The behavioral and brain sciences, v. 8, p. 567-616, 1985.

; BLOOM K. K. The alien hand sign. Localization, lateralization and recovery. American Journal of Physical Medicine \& Rehabilitation, v. 69, p. 228-238, 1990.

JESUS, D. B. The effect of 12 proficiency on the declarative and procedural memory systems of bilinguals: a psycholinguistic study. Dissertação (Mestrado em Letras/Inglês e Literatura Correspondente) - UFSC, Florianópolis, 2012.

LENT, R. Cem bilhões de neurônios? Conceitos fundamentais de neurociência. 2 ed. São Paulo: Editora Atheneu, 2010.

LUM, J. A.G.; CONTI-RAMSDEN, G.; PAGE, D.; ULLMAN, M. T. Working, declarative and procedural memory in specific language impairment. Cortex, v.48, p. 1138-1154, 2012.

MARR, D. Vision: a computational investigation into the human representation and processing of visual information. Massachussetts: W.H. Freeman and Company, 1982.

MINK, J. W.; THACH, W. T. Basal ganglia intrinsic circuits and their role in behavior. Current opinion in neurobiology, v. 3, p. 950-957, 1993.

MOLT, L. F. The basal ganglia's possible role in stuttering. Disponível em: http://www.mnsu.edu/comdis/isad2/papers/molt2.html. Acesso em 20 de junho de 2012.

NEMETH, D.; JANACSEK, K.; CSIFCSAK, G.; SZVOBODA, G.; HOWARD JR, J.H.; et al. Interference between sentence processing and probabilistic implicit sequence learning. PloS One, v. 6, p. 1-6, 2011.

KNOPMAN, D.; NISSEN, M. J. Procedural learning is impaired in Huntington's disease: evidence from the serial reaction time task. Neuropsychologia, v. 29, n. 3, p. 245-254, 1991.

OLIVEIRA, C. M. C. Instrumento de severidade da gagueira para crianças e adultos (RILEY, 1994) adaptado para o português brasileiro. Material didático do curso - Gagueira: avaliação, diagnóstico e terapia. Fundepe Cursos educação à distância, 2012.

PASSINGHAM, R. E. Two cortical systems for directing movement. Ciba Foundation Symposium, v. 132, p. 151-164, 1987.

PINKER, S. The language instinct. New York: William Morrow, 1994. 
RIASCOS, A. M. L.; CASTELLANOS, B. S. Aspectos del lenguaje en la enfermedad de Gilles de la Tourette: revisión sistemática de la literatura. Avances en Psicología latinoamericana, v. 29, n.2, p. 231-239, 2011.

RICHELS, C.; BUHR, A.; CONTURE, E.; NTOUROU, K. Utterance complexity and stuttering on function words in preschool-age children who stutter. Journal of fluency disorders, v. 35, p. 314-331, 2010.

RILEY, G. D. A stuttering severity instrument for children and adults. Austin: Pro-Ed, 1994.

SMITS-BANDSTRA, S.; DE NIL, L. F. Sequence skill learning in persons who stutter: Implications for cortico-striato-thalamo-cortical dysfunction. Journal of fluency disorders, v. 32, p. 251-278, 2007.

ULLMAN, M. T. The declarative/procedural model of lexicon and grammar. Journal of Psycholinguistic research, v. 30, p. 37-69, $2001 \mathrm{a}$.

. The neural basis of lexicon and grammar in first and second language: The declarative/procedural model. Bilingualism. Language and cognition, v. 4, p. 105-122, $2001 \mathrm{~b}$.

. A neurocognitive perspective on language: the declarative/procedural model. Neuroscience, v. 2, p. 717-727, 2001c

Contributions of memory circuits to language: The declarative/procedural model. Cognition, v. 92, p. 231-270, 2004.

The role of memory systems in disorders of language. Handbook of the Neuroscience of language.

Elsevier, p. 189-198, 2008.

; CORKIN, S.; COPPOLA, M.;HICKOK, G.; GROWDON, J. H.; et al. A neural dissociation within language: evidence that the mental dictionary is part of declarative memory, and that grammatical rules are processed by the procedural system. Journal of Cognitive Neuroscience, v. 9, p. 266-276, 1997.

; PIERPONT, E. L. Specific language impairment is not specific to language: the procedural deficit hypothesis. Cortex, v. 41, p. 399-433, 2005.

WALENSKI, M.; ULLMAN, M. T. The science of language. The Linguistic revierw, v. 22, p. 327-346, 2005.

Recebido em 12/07/2014. Aprovado em 28/09/14. 